\title{
Deuterium medicinal chemistry comes of age
}

\begin{abstract}
"With the advanced stage of development of the first wave of drug candidates and numerous additional deuterated agents progressing in the clinic, deuterium is now a validated component of the medicinal chemistry repertoire."
\end{abstract}

First draft submitted: 20 January 2016; Accepted for publication: 5 February 2016; Published online: 15 April 2016

Keywords: drug design $\bullet$ drug development $\bullet$ pharmacodynamics $\bullet$ pharmacokinetics

Deuterium is gaining increased appreciation and utilization as a component of active pharmaceutical ingredients. Deuteriummodified or -deuterated compounds retain the potency and selectivity of their hydrogen analogs. However, in certain cases, the deuterium isotope effect can significantly modify drug metabolism, offering the potential to positively impact safety, tolerability or efficacy properties [1-3]. Nevertheless, it has taken decades since deuterated drugs were first explored for them to reach late-stage clinical evaluation. Some earlier reviewers concluded that deuterium substitution would likely not significantly improve drugs and their commercialization was questionable or unlikely $[4,5]$. This editorial focuses on some recent advances in deuterium-modified drugs and discusses their unique development potential.

The use of deuterium to create a new chemical entity can in principle be approached in two ways. First, deuterium can be a tool in the traditional medicinal chemistry approach of iterative optimization in the de novo design of new compounds. Second, starting with an existing drug, hydrogen can be replaced by deuterium at specific sites without otherwise modifying the drug scaffold. These approaches are distinct with respect to drug development and regulatory approval. The US FDA has allowed sponsors of deuterated drugs to reference data of the hydrogen analogs in a 505(b)(2) application but not, to our knowledge, for any other modifications to the active moiety [6]. This regulatory advantage is currently unique to the modification of an existing drug with deuterium.

The first apparent substantial attempt to develop a deuterium-containing drug took the approach of utilizing deuterium to enable satisfactory properties in a novel molecular scaffold [7]. Since pharmaceutical companies have generally focused on developing novel chemotypes, this approach may likely continue as the more prevalent one in those institutions. The alternative approach, selectively incorporating deuterium as a targeted hydrogen replacement substituent in otherwise unchanged drug molecules, has been adopted by several companies such as Concert Pharmaceuticals and Auspex Pharmaceuticals (now a subsidiary of Teva Pharmaceuticals), and has seen considerable progress in recent years.

The recent success of deuterated drug candidates is the result of multiple converging factors that have helped to demonstrate their utility and likely economic viability:

- The FDA and EMA accepted, with supporting evidence, on a case-by-case basis, that deuterated compounds are highly similar to their hydrogen analogs with respect to interactions with biological receptors, leading to generally indistinguishable biochemical potency and selectivity. As a result, in several cases, sponsors developing deuterated analogs of approved drugs have been able to refer-

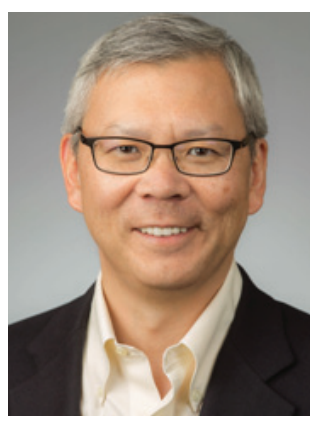

Roger D Tung Concert Pharmaceuticals, Inc., 99 Hayden Ave., Ste. 500, Lexington, MA 02421, USA

rtung@concertpharma.com 
ence data on the previously developed nondeuterated drug, reducing the cost and time necessary to support a submission package $[6,8]$.

- Even with substantial similarities to existing drugs, metabolic differences imparted by deuterium substitution have proven sufficient to enable clinically important differences relative to their hydrogen analogs. In the past year, deuterated analogs of approved drugs have been granted Fast Track, Orphan and Breakthrough Therapy Designation by the FDA $[6,9,10]$.

- Patent offices in numerous territories, including the USA, Europe, Japan and China, are granting patents on deuterated drugs, including composition of matter and method of use claims. A frequent basis of patentability is the unpredictability of the deuterium isotope effect, where the patent office determines that the differentiated compounds are nonobvious. The patentability of a deuterated compound was recently brought before a judicial body, which denied a request to invalidate a patent claiming deuterated venlafaxine [10].

- Detailed understanding of the individual contributions of a parent molecule and its metabolites to the therapeutic efficacy, safety, and tolerability of a given drug, which is currently possible only based on population pharmacokinetic and pharmacodynamic analyses, provides a rational basis for targeted metabolic modifications to improve clinical characteristics that can only be accomplished through deuterium incorporation.

- The cost of highly enriched $\mathrm{D}_{2} \mathrm{O}$ as a manufacturing feedstock is low enough to generally support a commercially acceptable cost of goods for manufacturing deuterated drugs.

- Advances in our understanding of human biology, as well as empirical clinical evidence from observational or investigator-initiated studies, provide continuing opportunities to deploy molecules possessing generally understood safety and efficacy parameters to new diseases. The potential for improved clinical properties and long-lived patent protection provide the justification to make the large investments necessary to develop deuterated drugs for new indications.

- In the past year, two companies which were largely or entirely focused on developing deuterium-containing drugs - Avanir Pharmaceuticals and Auspex - were each acquired for multibillion dollar sums. This tangible demonstration of the value of deuterated compounds is supportive of investments in both existing deuterated pipeline compounds and the identification of additional opportunities where deuteration of biologically active agents can create differentiated and valuable new drugs [11,12].

A number of deuterated compounds are currently undergoing clinical development, including the following:

AVP-786, a fixed-dose combination of $d_{6}$-dextromethorphan with an ultra-low dose of quinidine, is currently enrolling Phase III clinical trials for the treatment of agitation associated with Alzheimer's Disease dementia, an indication that currently has no FDA-approved treatments and for which there is a great unmet need [13]. Avanir had previously obtained approval for a combination product consisting of $20 \mathrm{mg}$ of dextromethorphan (nondeuterated) and $10 \mathrm{mg}$ of quinidine for a different indication [14]. The product Nuedexta ${ }^{\circledR}$ was approved in the USA and is marketed by Avanir for pseudobulbar affect, which is characterized by uncontrollable crying, laughing or other emotional displays occurring secondary to neurologic disease or brain injury. Quinidine is a metabolic inhibitor that enables the active ingredient dextromethorphan to reach therapeutic concentrations.

Avanir found that with AVP-786, due to its deuterium stabilization, a much lower amount of quinidine was needed to reach an equivalent plasma exposure of the active species - in this case, deuterated dextromethorphan. Due to the unmet need in Alzheimer's Disease-associated agitation, the FDA provided AVP786 with Fast Track designation [15]. Phase II clinical studies in treatment refractory major depression and residual schizophrenia are currently ongoing [13].

AVP-786 benefits from numerous worldwide patents, including composition of matter [16-18]. These patents on deuterated dextromethorphan and its combination with quinidine provide a period of exclusivity extending well beyond earlier patents protecting the nondeuterated drug combination.

SD-809, $d_{6}$-tetrabenazine, has completed two Phase III studies in the treatment of chorea due to Huntington's disease (sudden involuntary movements). Similar to deuterated dextromethorphan, deuterium incorporation markedly altered the pharmacokinetic behavior of tetrabenazine, increasing plasma half-life from 4.8 to $8.6 \mathrm{~h}$ and approximately doubling AUC exposure [19]. SD-809 ER, the clinical formulation of SD-809 used in its efficacy studies, adds extended release technology to further smooth out SD-809's pharmacokinetic curve. As a result of these stacked technologies, SD-809 is dosed less frequently than tetrabenazine, twice- versus three-times 
daily, and has a substantially smaller peak/trough ratio than does tetrabenazine [20].

SD-809 has been developed following the 505(b)(2) regulatory pathway for the same indication for which tetrabenazine is approved [6]. Top-line results released by Auspex from a placebo-controlled Phase III study suggest that the SD-809 ER possesses a much more benign side effect profile than does commercial tetrabenazine, presumably due to reduced peak-related adverse effects [20,21]. SD-809 was granted Orphan Drug Designation for SD-809. Its degree of clinical differentiation from commercial tetrabenazine was sufficient to support a Breakthrough Therapy Designation by the FDA, which accepted a New Drug Application for SD-809 in August, 2015 [8,9].

CTP-656, $\mathrm{d}_{9}$-ivacaftor, is currently being developed for the treatment of cystic fibrosis. Deuteration of ivacaftor provided unexpected results in that two isotopologs, respectively $\mathrm{d}_{9}$ and $\mathrm{d}_{18}$, while appearing similarly stabilized to hepatic metabolism in vitro, demonstrated differentiated pharmacokinetics from each other in vivo with differing rank order depending on the species studied. In rats, $\mathrm{d}_{18}$-ivacaftor provided greater exposure than did the $\mathrm{d}_{9}$-isotopolog, while in dogs the reverse was true. Both isotopologs were taken into a healthy volunteer crossover study, where $\mathrm{d}_{9}$-ivacaftor demonstrated greater human exposure than $\mathrm{d}_{18}$-ivacaftor.

In a subsequent single ascending dose Phase I study of CTP-656 that included a crossover comparison with

\section{References}

Papers of special note have been highlighted as:

- of interest

1 Baillie TA. The use of stable isotopes in pharmacological research. Pharmacol. Rev. 33(2), 81-132 (1981).

2 Harbeson SL, Tung RD. Deuterium in drug discovery and development. In: Annual Reports in Medicinal Chemistry. Macor JE (Ed.). Elsevier, Oxford, UK, 403-418 (2011).

3 Gant TG. Using deuterium in drug discovery: leaving the label in the drug. J. Med. Chem. 57(9), 3595-3611 (2014).

4 Fisher MB, Henne KR, Boer J. The complexities inherent in attempts to decrease drug clearance by blocking sites of CYPmediated metabolism. Curr. Opin. Drug Discov. Devel. 9(1), 101-109 (2006).

5 Foster AB. Deuterium isotope effects in the metabolism of drugs and xenobiotics: implications for drug design. In: Advances In Drug Research. Testa B (Ed.). Academic Press, London, UK, 14, 1-39 (1985).

6 Teva announces acceptance of SD-809 New Drug Application to the FDA.

www.tevapharm.com/news/teva_announces_fda

7 Darland GK, Hajdu R, Kropp H et al. Oxidative and defluorinative metabolism of fludalanine, 2-2H-3-fluoro-D- ivacaftor at $150 \mathrm{mg}$, CTP-656 provided about 3.5-fold greater AUC exposure than did ivacaftor, about a third longer plasma half-life and much greater exposure to the parent drug (the most active species) relative to metabolites than was observed with ivacaftor [22]. Concert Pharmaceuticals is planning to initiate efficacy studies in cystic fibrosis patients in 2016.

In addition, a growing number of other deuterated compounds have started clinical development or been evaluated as potential new drugs. These include, among others, CTP-730 (deuterated apremilast), JZP386 (deuterated sodium oxybate), CTP-499 (deuterated pentoxifylline metabolite), ALK-001 (deuterated vitamin A) and SD-560 (deuterated pirfenidone).

With the advanced stage of development of the first wave of drug candidates and numerous additional deuterated agents progressing in the clinic, deuterium is now a validated component of the medicinal chemistry repertoire.

\section{Financial \& competing interests disclosure}

The author is employed by Concert Pharmaceuticals and owns stock and stock options in the company. The author has no other relevant affiliations or financial involvement with any organization or entity with a financial interest in or financial conflict with the subject matter or materials discussed in the manuscript apart from those disclosed.

No writing assistance was utilized in the production of this manuscript.

alanine. Drug metabolism and disposition. 14(6), 668-673 (1986).

8 Avanir announces that AVP-923 data can be referenced in AVP-786 clinical development and submission. www.prnewswire.com/news-releases/avanir-pharmaceuticals

9 Auspex announces Breakthrough Therapy Designation for SD-809.

www.tevapharm.com/news/teva_announces_breakthrough

10 Neptune Generics, Llc V. Auspex Pharmaceuticals, Inc., U.S. Pat. 7456317, IPR2015-01313. http://fishpostgrant.com/wp-content/

11 Otsuka Pharmaceutical Completes Acquisition of Avanir Pharmaceuticals.

www.otsuka.com/en/hd_release/release/pdf.php? news $=1079$

12 Teva Completes Acquisition of Auspex Pharmaceuticals. www.tevapharm.com/news/teva_completes

13 Clinical Trials Search: AVP-786. https://clinicaltrials.gov/ct2/results?term =avp

14 Nuedexta ${ }^{\circledR}$ product label, FDA New Drug Application no. 021879 dated $01 / 20 / 2015$. www.accessdata.fda.gov/drugsatfda_docs/

15 Avanir announces Fast Track Designation for AVP-786. www.prnewswire.com/news-releases/avanir- 
16 Tung RD. US 7973049 B2 (2011).

17 Tung RD. US 8541436 B2 (2013).

18 Tung RD. US 7748450 B2 (2014).

- $\quad$ All patents for Morphinan compounds.

19 Stamler D, Bradbury M, Brown F. The pharmacokinetics and safety of deuterated-tetrabenazine. Neurology 80 (1), P07.210 (2013).

20 Auspex investor presentation at Jefferies 2014 Global Healthcare Conference.

www.jefferies.com/CMSFiles/Jefferies.com/files/
21 Xenazine $^{\circledast}$ product label, FDA New Drug Application no. 021894 dated 06/02/2015.

www.accessdata.fda.gov/drugsatfda_docs/label/

22 Harbeson SL, Nguyen S, Bridson G et al. Pharmacokinetic studies of deuterated isotopologs if ivacaftor in preclinical models and healthy volunteers. Presented at: 29th Annual North American Cystic Fibrosis Conference, Nashville, TN, USA, 8 October 2015.

www.concertpharma.com/wp-content/uploads 